\title{
THE STOMA REVERSAL AFTER HARTMANN PROCEDURE IN PATIENTS WITH COLORECTAL CANCER OPERATED IN EMERGENCY
}

\author{
Georgiana Bianca Constantin ${ }^{1}$, D. Firescu ${ }^{2,3}$, D. Voicu ${ }^{2}$, B. Ștefãnescu ${ }^{2,3}$, R. Mihailov ${ }^{1}$, Cristina \\ Șerban ${ }^{2,3}$, Rodica Bîrlă ${ }^{1,4}$, S. Constantinoiu ${ }^{1,4}$
}

${ }^{1}$ „Carol Davila” University of Medicine and Pharmacy, Bucharest, Romania

2,"Dunărea de Jos" University, Faculty of Medicine and Pharmacy, Galati, Romania

${ }^{3}$ Clinical Emergency County Hospital Sf. Ap. Andrei, Galati, Romania

${ }^{4}$, ,Sf. Maria” Clinical Hospital, Bucharest, Romania

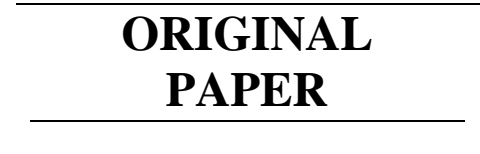

DOI: 10.33695/rojes.v2i1.21

Accepted: 22.03.2020

\footnotetext{
Corresponding author:

Georgiana Bianca

Constantin

constantin_bianca2009@ yahoo.com
}

\section{Abstract}

The Hartmann procedure still has an important place in the surgical therapy of complicated colorectal cancers. The morbidity rate can be very high and many patients never undergo the stoma reversal. We conducted a retrospective study on 431 patients with colorectal cancer operated in the second clinic of General Surgery of the Clinical Emergency County Hospital Galati between 2008-2017. $42,53 \%$ of the patients with Hartmann operation had a stoma reversal after a medium period of 5,7 months. The 5-years survival rate in patients with stoma reversal was $4,9 \%$. The reversal rate in our study is similar to those found in the literature in recent studies, that report percentages of $23,3 \%-55,17 \%$. In our study, the medium period between the emergency Hartmann procedure and the reversal of the stoma was 5,7 months, which is also similar to the data in the literature. We found a 5-years survival rate of $4,9 \%$ in patients with Hartmann reversal, much lower than others' results. Many stomas initially intended to be temporary, may not be reversed. It is important to optimize the timing of stoma closure. A period of approximately 6 months between the initial operation and the reversal seem to be adequate.

Keywords: colorectal cancer, emergency surgery, Hartmann, reversal

\section{Introduction}

Despite the screening possibilities, a lot of patients with colorectal cancer are still admitted in emergency, with complicated tumors, when the outcomes are poor. The Hartmann operation still has its place in the current surgical therapy and it is frequently used in the emergency management of complicated colorectal cancer, especially in patients with obstructed and/or perforated tumors.

The restoration of colonic continuity after emergency Hartmann procedure in patients with colorectal cancer can be technically challenging [1] and the timing of Hartmann's reversal is also debated. Stoma reversal can be associated with complications: wound infection, anastomotic leak, postoperative ileus, bowel obstruction, 
enterocutaneous fistula [2]. The morbidity rate can be very high (up to $50 \%$ ) and many patients never undergo the stoma reversal after Hartmann's procedure [3].

Regarding the timing, many authors suggest delaying the reversal by 3 months [4, 5] and others recommend waiting 6 months to allow the pelvic inflammation to resolve and the adhesions to decrease [6,7]. The risk factors for the stoma's non-closure are: advanced age, anastomotic leak, metastatic disease, adjuvant chemotherapy, significant comorbidities $[8,9,10]$.

The reversal technical procedure can be difficult, concerning the adhesiolysis, the identification of the rectal stump and the anastomosis itself [2]. The aim of this study is to evaluate the survival rate of the patients with stoma reversal after Hartmann procedure practiced in emergency conditions.

\section{Material and Method}

We made a retrospective observational study on 431 patients with complicated colorectal cancer, admitted and operated in the second clinic of General Surgery of the Clinical Emergency County Hospital Galati between 2008-2017. We studied the medical records, the histopathological reports and the surgery protocols.

Our working hypothesis was: the survival rate of the patients with stoma reversal after Hartmann procedure is much better than the survival rate of those patients who don't undergo the second intervention.

The inclusion criteria were: patients with malignant colorectal tumors; patients operated in emergency. We excluded the patients with benign colorectal pathology and those operated electively.

The statistical part of the study was made using Excel and SPSS programs. The probability of error less than $5 \%(\mathrm{p}<0.05)$ was considered the significance threshold.

\section{Results}

In 60 cases $(13,92 \%)$, the tumors were located on the cecoascendant colon and right flexure, in 30 cases $(6,98 \%)$ on the transverse colon, $57(13,22 \%)$ on the descendant colon and left flexure, $133(30,85 \%)$ on the sigmoid colon and $151(35,03 \%)$ were rectal tumors (Figure 1).

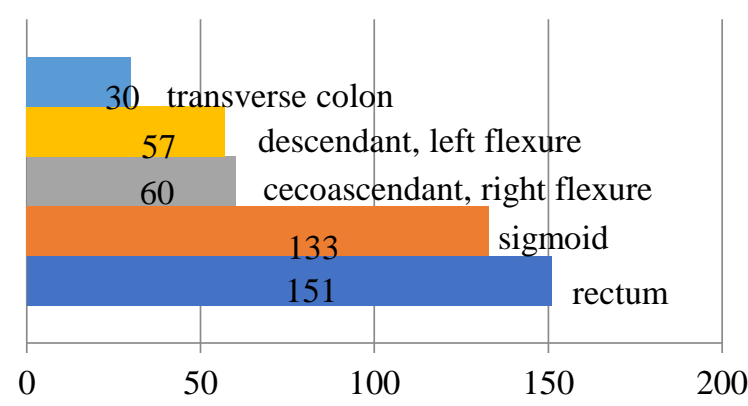

Figure 1 - The distribution of cases according the the location of the tumors

There have been practiced: 179 (41,5 $\%$ ) external derivations (158 colostomies and 21 Pezzer cecostomies), $134 \quad(31,1 \%)$ Hartmann operations, 89 (20,9\%) resections with anastomosis and 29 internal derivations (Figure 2).

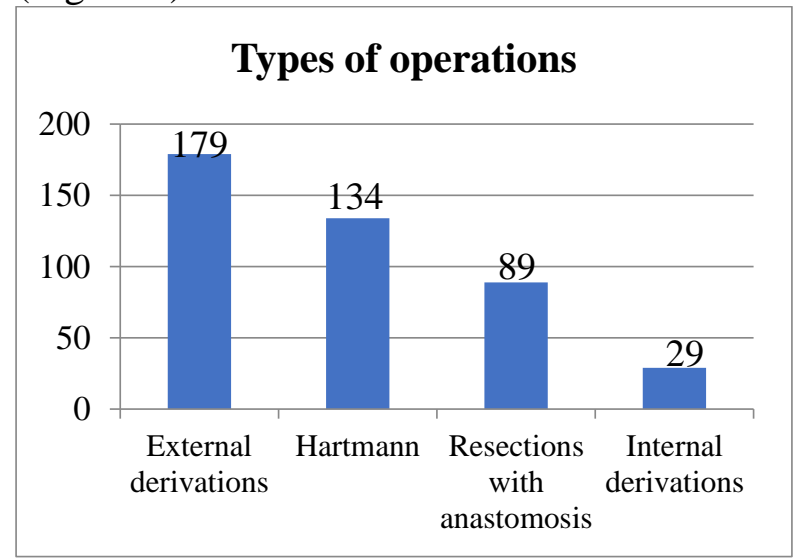

Figure 2 - The repartition of cases according to the type of surgery in emergency

Regarding the patients with Hartmann procedure, $57(42,53 \%)$ had a stoma reversal in order to reestablish the colon continuity. None of the patients with stoma reversal 
developed postoperatory complications. The reversal has been made after a medium period of 5,7 months.

49 of these 57 patients died and only 8 survived until 01.10.2019 (the date when this study ended). None of the patients without stoma reversal survived. Comparing the survival rate in the 2 groups, we obtained statistically significant difference (p_value < 0,00001 Log Rank (Mantel-Cox)) (Figure 3).

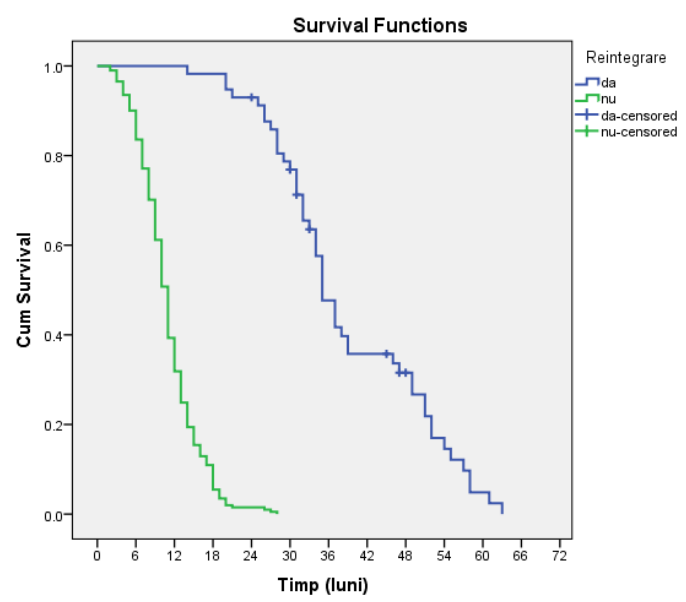

Figure 3 - Survival curves, Kaplan-Meier test according to stoma reversal

\begin{tabular}{|c|c|c|c|c|c|c|c|c|}
\hline \multirow[t]{3}{*}{ Reversal } & \multicolumn{4}{|c|}{ Mean } & \multicolumn{4}{|c|}{ Median } \\
\hline & \multirow[t]{2}{*}{ Estimate } & \multirow[t]{2}{*}{$\begin{array}{l}\text { Std. } \\
\text { Error }\end{array}$} & \multicolumn{2}{|c|}{$\begin{array}{l}\text { 95\% Confidence } \\
\text { Interval }\end{array}$} & \multirow[t]{2}{*}{ Estimate } & \multirow[t]{2}{*}{$\begin{array}{l}\text { Std. } \\
\text { Error }\end{array}$} & \multicolumn{2}{|c|}{$\begin{array}{l}\text { 95\% Confidence } \\
\text { Interval }\end{array}$} \\
\hline & & & $\begin{array}{l}\text { Lower } \\
\text { Bound }\end{array}$ & $\begin{array}{l}\text { Upper } \\
\text { Bound }\end{array}$ & & & $\begin{array}{l}\text { Lower } \\
\text { Bound }\end{array}$ & $\begin{array}{l}\text { Upper } \\
\text { Bound }\end{array}$ \\
\hline Yes & 39.156 & 1.686 & 35.852 & 42.461 & 35.000 & 1.303 & 32.446 & 37.554 \\
\hline No & 10.965 & .325 & 10.328 & 11.603 & 11.000 & .315 & 10.383 & 11.617 \\
\hline Overall & 17.191 & .856 & 15.513 & 18.868 & 12.000 & .462 & 11.094 & 12.906 \\
\hline
\end{tabular}

Table 1 - The mean and median estimated time of survival according to stoma reversal

\begin{tabular}{|l|l|l|l|l|l|l|}
\hline Survivors & 6 months & 12 months & 18 months & 24 months & 36 months & 60 months \\
\hline Yes & $\mathbf{1 0 0 \%}$ & $\mathbf{1 0 0 \%}$ & $\mathbf{9 8 . 2 \%}$ & $\mathbf{9 3 . 0 \%}$ & $\mathbf{4 7 . 7 \%}$ & $\mathbf{4 . 9 \%}$ \\
\hline No & $\mathbf{8 3 . 6 \%}$ & $\mathbf{3 1 . 8 \%}$ & $\mathbf{5 . 5 \%}$ & $\mathbf{1 . 5 \%}$ & $\mathbf{0 . 0}$ (Maximum 28) & - \\
\hline
\end{tabular}

Table 2 - The 5-years survival rate according to stoma reversal

There are authors who consider that the emergency Hartmann procedure for left-sided colorectal cancer is an obsolete operation, often performed only with palliative intention, in elderly patients, with significant comorbidities, with a high risk of intercurrent
The mean-estimate time of survival for patients with stoma reversal is 39,156 and for those without stoma reversal is 10,965 . The median-estimate time of survival (the time in which the event happens in $50 \%$ of the cases) for patients with stoma reversal is 35,000 and for those without stoma reversal 11,000 (Table 1). The 5-years survival rate in patients with stoma reversal is $4,9 \%$ and $0 \%$ for those who didn't undergo the reversal (Table 2).

\section{Discussions}

In July 2016, the Scientific Board of the World Society of Emergency Surgery prepared a possible consensus focusing on the emergency colorectal surgery and presented their work during the congress that has been held in Campinas, Brazil in 2017. They have only agreed that the Hartmann operation should be the treatment of choice in left-sided perforations, but the emergency surgical treatment for obstructed colorectal cancer is still debated [11]. 
The reversal rate in our study is 42,53 $\%$. We found in the literature a lot of studies that assessed the safety of Hartmann's procedure in terms of postoperative morbidity and mortality and long-term survival. In a study from 1981, the reversal rate after Hartmann procedure was only $9,34 \%$ [14]. In more recent studies, the reversal rate is much higher: $23,3 \%-55,17 \%$ [15, 16, 17]. A recent Romanian study that included 590 patients operated in Cluj-Napoca between 1996-2005, report a reversal rate of $68 \%$ [18].

In our study, the medium period between the emergency Hartmann procedure and the reversal of the stoma was 5,7 months. In other studies, the Hartmann's reversal in patients with colorectal cancer is usually delayed for 8-10 months, but not more than that. The experience from the Dutch Rectal Cancer Trial has shown that if a stoma was not closed within the first year, it would probably become permanent [10].

We found a 5-years survival rate of 4,9 $\%$ in patients with Hartmann reversal, much lower than others' results. According to the recent EUROCARE 5 analysis, colon and rectal cancer presented a minimal but significant increase in 5-year survival [19]. Kristiansen reported a 5-year survival rate of $31 \%$ and that intestinal continuity was restored in $7(24 \%)$ of 29 patients who underwent Hartmann operations for obstructive left-sided colorectal cancers [20].

\section{Conclusions}

The Hartmann procedure is a fast and safe intervention in emergency surgery for colorectal cancers, that allows the resection of the tumor, avoiding in the same time the anastomotic fistulas. Many stomas initially intended to be temporary, may not be reversed due to different factors, such as: advanced age, postoperative complications, adjuvant therapy and prohibitive comorbidities.
It is important to optimize the timing of stoma closure. A period of approximately 6 months between the initial operation and the reversal seems to be adequate.

\section{References}

[1] Zachariah S K. Reverse transrectal stapling technique using the EEA stapler: an alternative approach in difficult reversal of Hartmann's procedure. J Surg Tech Case Rep. 2010;2(02):70-72

[2] Sherman KL, Wexner SD. Considerations in Stoma Reversal. Clin Colon Rectal Surg. 2017;30(3):172-177

[3] Bell C, Asolati M, Hamilton E et al.A comparison of complications associated with colostomy reversal versus ileostomy reversal. Am J Surg. 2005;190(05):717-720

[4] Di Carlo I, Toro A, Pannofino O, Patane E, Pulvirenti E. Laparoscopic versus open restoration of intestinal continuity after Hartmann procedure. Hepatogastroenterology. 2010;57(98):232235

[5] Keck J O, Collopy B T, Ryan P J, Fink R, Mackay J R, Woods R J. Reversal of Hartmann's procedure: effect of timing and technique on ease and safety. Dis Colon Rectum. 1994;37(03):243-248

[6] Slawik S, Dixon A R. Laparoscopic reversal of Hartmann's rectosigmoidectomy. Colorectal Dis. 2008;10(01):81-83

[7] Fleming F J, Gillen P. Reversal of Hartmann's procedure following acute diverticulitis: is timing everything? Int J Colorectal Dis. 2009;24(10):1219-1225

[8] Gessler B, Haglind E, Angenete E. Loop ileostomies in colorectal cancer patients-morbidity and risk factors for nonreversal. $\mathrm{J}$ Surg Res. 2012;178(02):708-714

[9] Lindgren R, Hallböök O, Rutegård J, Sjödahl R, Matthiessen P. What is the risk for a permanent stoma after low anterior resection of the rectum for cancer? A six-year 
follow-up of a multicenter trial. Dis Colon Rectum. 2011;54(01):41-47

[10] den Dulk M, Smit M, Peeters K C et al.A multivariate analysis of limiting factors for stoma reversal in patients with rectal cancer entered into the total mesorectal excision (TME) trial: a retrospective study. Lancet Oncol. 2007;8(04):297-303

[11] Pisano, M., Zorcolo, L., Merli, C. et al. 2017 WSES guidelines on colon and rectal cancer emergencies: obstruction and perforation. World J Emerg Surg 2013, 36 (2018)

[12] Meyer F, Marusch F, Koch A, Meyer L, Fuhrer S, Köckerling F, Lippert H, Gastinger I, the German Study Group "Colorectal Carcinoma (Primary Tumor)": Emergency operation in carcinomas of the left colon: value of Hartmann's procedure. Tech Coloproctol. 2004, 8: S226-229

[13] Charbonnet, P., Gervaz, P., Andres, A. et al. Results of emergency Hartmann's operation for obstructive or perforated leftsided colorectal cancer. World J Surg Onc 2008 (6): 90

[14] ReMine SG, Dozois RR. Hartmann's Procedure: Its Use With Complicated Carcinomas of Sigmoid Colon and Rectum. Arch Surg. 1981;116(5):630-633

[15] David GG, Al-Sarira AA, Willmott S, et al. Use of Hartmann's procedure in England. Colorectal Dis 2009; 11:308-12
[16] Pearce NW, Scott SD, Karran SJ. Timing and method of reversal of Hartmann's procedure. Br J Surg 1992;79:839-41

[17] Roque-Castellano C, Marchena-Gomez J, Hemmersbach-Miller M, Acosta-Merida A, Rodriguez-Mendez A, Fariña-Castro R, Hernandez-Romero J: Analysis of the factors related to the decision of restoring intestinal continuity after Hartmann's procedure. Int J Colorectal Dis. 2007,22:1091-1096

[18] F. Zaharie, L. Mocan, T. Mocan, C. Tomus, V. Hodor, N. Al Hajjar, D. Munteanu, I. Puia, O. Bala, R. Zaharie, O. Ilie, D. Bartos, A. Bartos, L. Vlad, C. Iancu. Managementul chirurgical în urgență al ocluziilor prin cancere colonice și rectale. Chirurgia 2011,106(4):479-484

[19] Holleczek B, Rossi S, Domenic A ,et al; EUROCARE-5 Working Group. Ongoing improvement and persistent differences in the survival for patients with colon and rectum cancer across Europe 1999-2007 Results from the EUROCARE-5 study .Eur $J$ Cancer;2015 .S0959-8049(15)00704-2

[20] Kristiansen VB, Lausen IM, Frederiksen HJ, Kjaergaard J: Hartmann's procedure in the treatment of acute obstructive left-sided colonic cancer. Ugeskr Laeger. 1993,155:3816-3818 